\title{
DERECHO A LA CIUDAD Y UNA EXPERIENCIA EDUCATIVA POPULAR
}

\author{
Marilín López Fittipaldi ${ }^{1}$
}

\section{Presentación}

La ciudad de Rosario (Argentina) ${ }^{2}$ ha atravesado en las últimas décadas una serie de transformaciones que reconfiguran la trama urbana y hacen emerger nuevas conflictividades. La puesta en valor de determinados espacios de la ciudad a través de proyectos que articulan al sector público y privado se acompañó de la profundización del deterioro de las condiciones de vida de los sectores más pobres.

Segregación espacial (Broguet, 2016), profundización de los procesos de violencia en vinculación con los circuitos delictivos y del narcotráfico (Cámpora et al., 2017), y falta de acceso a derechos básicos en las periferias son algunos de los aspectos que marcan la contracara de un imaginario de ciudad turística que se fue imponiendo en los últimos años (Vera, 2012).

Pero al mismo tiempo, como contrapartida de estos procesos, emergen sujetos colectivos que hacen de la ciudad escenario y objeto de lucha, convirtiéndose en actores significativos en la configuración del espacio urbano. En este trabajo nos proponemos, entonces, enfocar el proyecto político-educativo de un movimiento social en el contexto de la lucha por el derecho a la ciudad (Harvey, 2014).

Las reflexiones que sustentan este trabajo surgen a partir de una línea de investigación que, desde un enfoque antropológico relacional, venimos desarrollando en el campo de los movimientos sociales urbanos ${ }^{3}$. Nuestro interés se ubica en torno a

\footnotetext{
${ }^{1}$ Universidad Nacional de Rosario, Argentina.

${ }^{2}$ Rosario es una ciudad ubicada en el centro del país, sobre las orillas del río Paraná, en el suroeste de la provincia de Santa Fe. De acuerdo a los datos del Censo Nacional de Población y Viviendas 2010 cuenta con una población de 948.312 habitantes, siendo, en relación a su población, la tercera ciudad de Argentina y la mayor ciudad de la provincia (Fuente: https://www.rosario.gov.ar /web/ciudad/caracteristicas/informacion-territorial-y-datos-demograficos).

${ }^{3}$ Nuestro trabajo de investigación en torno a un movimiento social de la ciudad de Rosario se inicia, de modo exploratorio, en el año 2010. A partir del año 2011 nos centramos en el proyecto político-educativo impulsado por la organización en un barrio periférico de la ciudad. Como resultado de esta primera etapa de trabajo, hemos elaborado la tesis de Licenciatura "Movimientos sociales y educación. Un análisis antropológico del proceso de construcción de un 'Bachillerato Popular' en la ciudad de Rosario" (año 2015). Actualmente, hemos iniciado una segunda etapa de indagación, a partir del Proyecto de Doctorado "Movimientos sociales, jóvenes y educación. Un análisis antropológico de los "Bachilleratos Populares" como experiencias socioeducativas emergentes en contextos de desigualdad social". El trabajo de campo,
} 
experiencias socioeducativas que emergen como parte de organizaciones políticas y/o movimientos sociales en entramados barriales de pobreza urbana y desigualdad social. Específicamente, nos enfocamos en escuelas para jóvenes y adultos, que en la Argentina han sido usualmente denominadas "Bachilleratos Populares"4.

A partir de su primera aparición, los "Bachilleratos Populares" se replicaron fundamentalmente en la Capital Federal y en provincia de Buenos Aires, aunque también se irradiaron, con menos fuerza, a otras provincias del país 5 . De modo concomitante, también se multiplicaron las investigaciones que buscaron estudiarlos. Así, es posible encontrar trabajos que han puesto de relieve distintas dimensiones de análisis para su comprensión, desde las características de la propuesta educativa (Langer, 2011; Gluz, 2013) hasta las relaciones que entablan con el Estado en la búsqueda de reconocimiento (García, 2011; Caisso, 2014). También se ha destacado como dimensión significativa la relación con el territorio, o con lo barrial, enfocando las estrategias educativas como parte de esa trama social (Elisalde, 2008), y señalado "la inmanencia de la propuesta pedagógica con el espacio social y sus demandas" (Ampudia, 2013: 48) . $^{6}$

Nos interesa, en este escrito, detenernos en este último punto. ¿Qué característica adquiere, en su concreción, la relación entre la experiencia educativa y la trama barrial en la que se inscribe?

desarrollado entre los años 2010 y 2013 y reiniciado en el 2016, se basa en observaciones, entrevistas informales y en profundidad, así como relevamiento de información periodística y de documentos del movimiento social. En términos teóricos y metodológicos, este trabajo se inscribe en un enfoque antropológico relacional, lo que supone un énfasis en las prácticas, sentidos y relaciones que construyen los sujetos cotidianamente, en su interrelación con procesos institucionales y estructurales más amplios.

${ }^{4}$ Los denominados "Bachilleratos Populares" hacen su primera aparición en el año 2004 en la Ciudad de Buenos Aires, y posteriormente comienzan a irradiarse a otros lugares del país. Se trata de escuelas secundarias para jóvenes y adultos impulsadas y gestionadas por movimientos sociales, empresas recuperadas y organizaciones sociales y políticas de distinto tipo que luego de su creación y con distinto éxito, han luchado por obtener el "reconocimiento oficial". El mismo supone, en general, la posibilidad de otorgar títulos que certifiquen el cumplimiento de la educación secundaria, y en ciertos casos, algún tipo de financiamiento estatal para el proyecto educativo.

5 A partir de un relevamiento a nivel nacional, el GEMSEP (Grupo de Estudios Sobre Movimientos Sociales y Educación Popular) estableció la existencia de 89 experiencias educativas de este tipo, distribuidas principalmente en Capital Federal y provincia de Buenos Aires, así como también en las provincias de Santa Fe y Mendoza (Carnelli, Rubinsztain, Said, 2015). Solo tres de ellos se ubican la provincia de Santa Fe, de los cuales dos están emplazados en la ciudad de Rosario.

${ }^{6}$ De hecho, esto no remite a una característica que se circunscribe solo a los denominados "Bachilleratos Populares". En este sentido, Santillán destaca que las demandas de los sectores populares y los movimientos sociales por el derecho a la educación adquieren, en las últimas décadas, "sentidos fundamentalmente territorializados. Esto es, las demandas se inscriben de lleno en los barrios y los escenarios cotidianos de vida de sus destinatarios" (Santillán, 2015: 2). 
La experiencia educativa que es foco de nuestra investigación se puso en marcha en 2011, siendo la primera de este tipo en la ciudad y en la provincia de Santa Fe. Si bien recuperaba la influencia que iban ejerciendo los "Bachilleratos Populares" a nivel nacional, rápidamente iba a adquirir características propias, fuertemente ligadas al derrotero del proyecto político del movimiento social que la puso en marcha ${ }^{7}$. El mismo había surgido a partir de la iniciativa de un grupo de jóvenes, en su mayoría estudiantes universitarios, que se propusieron desarrollar actividades educativas con niños en un barrio de la zona noroeste de la ciudad de Rosario. Las inundaciones y los desalojos, problemáticas que marcaban las condiciones de vida en ese contexto de la periferia urbana, rápidamente se convirtieron en eje de las demandas que comenzaban a formularse al gobierno local. La denominada "lucha por la tierra" vertebraría el proyecto político de la incipiente organización y marcaría el ritmo de su actividad territorial $^{8}$.

De este modo, aún sin desconocer la especificidad de las reivindicaciones ligadas al campo educativo, proponemos comprender la creación de escuela del movimiento social a la luz de la lucha por el derecho a la ciudad. Sostenemos que, en vinculación a esta demanda, se desplegaron procesos de producción colectiva del espacio urbano, en los que se inscribe la puesta en marcha de la experiencia escolar.

Con este objetivo, en este trabajo partimos considerando algunos planteos que dan cuenta de las transformaciones del espacio en el contexto neoliberal (Brenner y Theodore, 2002; Moctezuma, 2016) que ponen a la ciudad como foco de absorción de capital excedente a partir del desarrollo urbano (Harvey, 2014). Dentro de esta trama apuntamos a comprender los procesos que dieron forma a la periferia de la ciudad de Rosario, y específicamente, al contexto barrial en el que se desarrolla el movimiento

\footnotetext{
${ }^{7}$ En el momento de su creación, los militantes del movimiento social afirmaban que se trataba de "el primer Bachillerato Popular de la provincia de Santa Fe", y que surgía "inspirado en una experiencia muy interesante que se dio en la provincia de Buenos Aires (...) con este nombre de Bachilleratos Populares" (R12. Militante del movimiento social. Observación en acto de inauguración de la escuela. Fecha: 04/04/2011). Posteriormente, al calor del proceso de negociación por el reconocimiento oficial de la escuela y de las distintas reformulaciones que iba atravesando el proyecto político de la organización, los militantes y docentes enfatizaron la diferencia con los "Bachilleratos Populares" y resaltaron las semejanzas con las escuelas oficiales de la provincia. Argumentaba una de las docentes: "la diferencia [con los "Bachilleratos Populares"] tiene que ver, básicamente con una cuestión de estructura curricular, y con profundidad de contenidos. Los bachi tienen una estructura bastante flexible, con un formato más tipo taller (...) La nuestra es bastante más académica, (...) la estructura de materias y los programas, son como cualquier secundaria para adultos" (R. 43. Docente. Entrevista. Fecha: 24/05/13).

${ }^{8}$ Años más tarde, la organización política definiría la conformación de un partido político que ampliaría su accionar a escala de la ciudad. Sin embargo, aquí acotamos el análisis al momento de creación y consolidación de la experiencia educativa, por lo que nos limitamos a considerar las dimensiones ligadas a la lucha territorial del movimiento social y su anclaje barrial.
} 
social. Posteriormente, enfocamos los procesos de demanda que impulsó la organización en relación a ciertas problemáticas barriales, desde una perspectiva no dicotómica sobre los movimientos sociales urbanos (Evers y otros, 1982), ubicando las condiciones de vida en las periferias urbanas como base de procesos de politización y movilización (Holston, 2009) . En este contexto, la reivindicación del derecho no solo a consumir, sino fundamentalmente a producir la ciudad, será la clave desde la que intentaremos comprender la creación de la escuela del movimiento social.

\title{
La ciudad y su periferia: urbanización, expropiación y despojo
}

\begin{abstract}
Esos terrenos quieren convertirlos en un country, entonces constantemente se quiere desalojar a los vecinos. Vienen de a uno, y les ofrecen plata, o les dicen que los van a sacar con topadoras, y algunos se terminan yendo. Entonces pusimos las huertas ahí, para que vean actividad, movimiento... Y con esta producción se fue cambiando el reclamo, porque si los desalojan los vecinos no pierden solo la casa, sino también la tierra con la que producen. El reclamo ahora es por la vivienda y la tierra (R2. Militante. Entrevista y recorrida por el barrio. Fecha: 21/05/2010).
\end{abstract}

En la actualidad, resulta de común acuerdo que el futuro del planeta será urbano: las estimaciones de Naciones Unidas para el año 2050 indican que dos tercios de la población mundial residirá en ciudades. El consecuente interés por la problemática urbana se ha orientado, frecuentemente, a los fenómenos vinculados a las imponentes megalópolis, dejando en vacancia el conocimiento acerca de las ciudades de menor tamaño, las denominadas ciudades medias ${ }^{9}$.

Preguntarse por la ciudad es, a la vez, preguntarse por la concentración geográfica y social de un excedente en la producción que es inherente a la ciudad desde su origen (Harvey, 2014). Inicialmente a partir de la transferencia de excedentes del campo a la ciudad, luego como apropiación interna del espacio urbano, a través del uso

\footnotetext{
${ }^{9}$ Existen distintos criterios para definir a las ciudades de escala media, un conjunto que agrupa realidades ampliamente heterogéneas. Si bien los criterios cuantitativos oscilan, se puede tomar como referencia un rango poblacional entre los 50.000 y cerca del millón de habitantes (Gravano, 2014). Por otro lado, variables de orden cualitativo significativas refieren a su rol de "intermediación" entre las ciudades mayores y los espacios rurales y la prestación de servicios a localidades menores articuladas en una red regional (Punsola, 2014). También se ha destacado la importancia de considerar aspectos contextuales y "las dinámicas simbólicas que constituyen los entramados socio-históricos específicos" (Silva, 2016: 130). En el caso de la ciudad de Rosario, mientras que por su población -de un millón de habitantespodría considerarse en el límite del rango medio, si la consideramos contextualmente se cuenta entre las mayores del país. Por otro lado, como veremos, resulta importante atender a imaginarios -plasmados en las políticas públicas- que la ubican como una ciudad en transición, que abandona la escala intermedia para convertirse en una gran metrópoli. En este escenario, se despliegan transformaciones y reordenamientos que ponen al proyecto de ciudad en el centro de la contienda política.
} 
del espacio en forma asimétrica y desigual (Gravano, 2013), la ciudad presupone siempre relaciones de dominación y explotación (Singer, 1975).

Las ciudades han ocupado un rol estratégico en el re-hacerse de los espacios económico-políticos en la etapa del "neoliberalismo realmente existente" (Brenner y Theodore, 2002). En este contexto, señalan Brenner y Theodore, las ciudades y las periferias suburbanas del capitalismo avanzado se han convertido en laboratorios para una variedad de políticas neoliberales, que se orientan a la producción de la ciudad como un espacio para el crecimiento económico y las prácticas de consumo de las elites. Bajo estas condiciones, argumentan, las ciudades se han transformado en el espacio de incubación de las estrategias políticas e ideológicas a través de las cuales la dominación del neoliberalismo se mantiene, pero también, en sitios prominentes para la lucha sociopolítica (Brenner y Theodore, 2002).

Al mismo tiempo, algunos autores han llamado la atención sobre la importancia de dar cuenta de la significación y especificidad de los procesos recientes de transformación urbana en Latinoamérica (Janoschka y Sequera, 2014; Moctezuma, 2016). Así, autores como Moctezuma (2016) han apuntado a formular una "reinterpretación situada" a partir del contexto latinoamericano y del trabajo antropológico que permita mostrar la relación entre la renovación y revalorización económica y los desplazamientos y exclusiones sociales en un contexto de transformación del espacio neoliberal.

De acuerdo con Janoschka y Sequera (2014), las políticas urbanas implementadas durante los últimos años en la mayoría de las ciudades latinoamericanas - aun cuando existen diferencias entre los distintos lugares - apuntan a una transformación del espacio urbano que desencadena el desplazamiento progresivo de los sectores sociales de bajos recursos y, en articulación con la inversión inmobiliaria, la recuperación de los espacios centrales por las capas medias y altas. Este proceso, denominado gentrificación en el mundo anglosajón, se caracteriza en este contexto por el rol decisivo que juegan las administraciones públicas en relación a la creación de nuevos mercados inmobiliarios, y el carácter especialmente violento de los desplazamientos y de la formalización de economías y ciudades producidas y vividas en gran medida bajo la informalidad (Janoschka y Sequera, 2014). 
Al enfocar la ciudad de Rosario, donde desarrollamos nuestra investigación, encontramos que no constituye de ningún modo una excepción a estos procesos de alcance regional. En las últimas décadas, se pusieron en marcha distintos proyectos de planificación estratégica que fueron paulatinamente apuntando a transformar la ciudad. En este contexto, el Plan Urbano Rosario 2007/2017 se constituyó en una plataforma que orientó el proceso de desarrollo y transformación urbana a partir de ciertas premisas, que recogen imaginarios urbanos hegemónicos ${ }^{10}$. En dicho Plan, la ciudad es concebida a partir de aquello que deja de ser, para entrever en qué se quiere convertir: "el núcleo de escala intermedia se va convirtiendo paulatinamente en una metrópoli que amplía y diversifica su oferta de actividades y servicios para una extensa región" (PUR 2007/2017. El subrayado es nuestro) ${ }^{11}$.

La profunda transformación prevista e impulsada por el gobierno local se apoya, según el PUR, en dos pilares fundamentales: la planificación y la concertación con el sector privado, invocado como actor necesario en la producción del espacio público, y para hacer realidad una imagen de ciudad vinculada a la recreación, al ocio, y especialmente, al turismo (Vera, Roldán y Pascual, 2015). Pero al mismo tiempo, este proceso de conversión y crecimiento conlleva la emergencia de tensiones y conflictividades. En el propio PUR se reconoce "el problema de la pobreza, la exclusión y el crecimiento de las áreas de borde de la planta urbana" (PUR 2007/2017) como un gran desafío, que requiere atención prioritaria.

De este modo, mientras el centro de la ciudad y las áreas costeras se reconvierten para su aprovechamiento por las capas medias, los sectores acomodados y el crecientemente incentivado turismo, se profundizan procesos de reordenamiento espacial que van ubicando a los sectores más desfavorecidos en los márgenes de la ciudad, dando como resultado una "concentración geográfica de la pobreza" (Auyero, 2001, citado en Bernardi y Sanchez, 2006). Una concentración que se vio reforzada por proyectos públicos de construcción de conjuntos habitacionales destinados a la relocalización de asentamientos irregulares que fueron progresivamente extendiendo la planta urbana (Cámpora y Giampani, 2006). A la vez, como parte de un proceso que no es univoco, las zonas periféricas de la ciudad también se han vuelto foco de

\footnotetext{
${ }^{10}$ Como señala Paula Vera, el análisis de los imaginarios urbanos que emergen de las políticas del gobierno local adquiere relevancia en tanto "las significaciones sociales que generan cumplen las funciones tanto de instituir o crear como de mantener el orden social existente" (2013: 157)

${ }^{11}$ PUR 2007/2017. Consultado el 20 de septiembre de 2017 en: http://www.rosario.gov.ar
} 
emprendimientos urbanísticos de carácter privado, orientados a la construcción de espacios habitacionales de carácter exclusivo.

En el extremo noroeste de esta periferia urbana en conflicto se ubica el barrio en donde nace el movimiento social. En el pasado, hace poco más de un siglo, era una zona de quintas, caracterizada por la producción tambera, la elaboración artesanal de ladrillos, y una fábrica de materiales cerámicos. Su crecimiento inicial estuvo ligado también al ferrocarril, cuyas vías -hoy reemplazadas por una ruta provincial- demarcan el lado "este" y el lado "oeste", diferenciando "dos barrios" de los que ya casi no se recuerda su origen común (Nemcovsky, 2004).

En la década del setenta, la empresa dedicada a la producción de cerámicos cedió al Servicio Público Municipal de Vivienda terrenos para la construcción de complejos habitacionales, que fueron posteriormente acompañados por la radicación de asentamientos precarios (De Marco, 2016). Con el tiempo, la urbanización se fue extendiendo hacia el oeste, que si bien, recuerdan los habitantes de la zona, fue el origen fundacional del barrio, luego había quedado relegado ante el crecimiento del lado este, de tierras más altas (Nemcovsky, 2004).

Junto a las casas de material, antiguas, cercanas a las instalaciones del ferrocarril, fue creciendo el barrio hacia el oeste, hasta la "frontera" que marca un canal que corre casi en paralelo, a unos 800 metros del trazado de la vía. Luego del canal, como último cordón, un asentamiento que es caracterizado como de tipo rural, con viviendas de construcción precaria, y donde muchas familias emprenden la producción artesanal de ladrillos y cierta producción de subsistencia: cría de pequeños animales y/o el cultivo de huertos domésticos.

De acuerdo con la información que hemos podido recabar, en ciertos casos esta producción permite complementar la reproducción del grupo familiar, junto a una diversidad de actividades más o menos formales - trabajo en relación de dependencia y distintas formas de cuentapropismo - o decididamente informales - "changas", "cirujeo" 12 - En algunos casos -aunque es necesario profundizar la indagación- las familias se trasladan a este sector por temporadas, para luego retornar a zonas más "urbanizadas". Por este motivo, es posible sostener que esta "ruralización de lo urbano" (Evers y otros, 1982) se vincula directamente con la insuficiencia del trabajo remunerado para satisfacer las necesidades básicas para la reproducción de la población

\footnotetext{
${ }^{12}$ En Argentina, el término "changas" refiere a trabajos informales, esporádicos, y/o intermitentes. El "cirujeo" es la búsqueda y recolección de residuos que puedan ser reutilizados o vendidos.
} 
que vive en este lugar. En este espacio "poroso" se va difuminando gradualmente la distinción entre la ciudad y el campo (Harvey, 2014), a medida que el proceso de autourbanización avanza sobre los márgenes de la ciudad, pero conservando ciertos niveles de producción de tipo rural.

Los habitantes de la zona no tienen garantizado el acceso a los servicios urbanos tales como la energía eléctrica y el agua potable. Dentro del primer cordón -hacia el este del canal- el tendido de red solo llega hasta la calle principal, y desde allí los habitantes han extendido el mismo hasta sus domicilios. Hacia el oeste del canal, por otro lado, no hay provisión alguna de servicios, solo agua potable en cubas. A su vez, el desborde frecuente del canal y la baja altura de los terrenos aledaños convertieron a esta amplia zona en inundable, considerada en consecuencia por el municipio como "rural" y "no urbanizable". Ello permitió sostener su ocupación irregular por décadas, dando lugar a la existencia de un mercado informal de venta y alquiler de terrenos y viviendas. De este modo, la producción del espacio urbano se dio a partir de la ocupación de terrenos y la auto-provisión de servicios públicos, es decir, a partir de "formas autogestivas de acceso a la ciudad" (Cravino, 2015: 135).

Sin embargo, la falta de interés inmobiliario era solo aparente. Una vez efectuadas las obras públicas que reducen el impacto de las inundaciones en la zona, salió a la luz un proyecto de construcción de un barrio privado que ya se estaba llevando adelante, comprando terrenos a precios bajísimos y desalojando a vecinos que por décadas habían habitado allí. Estas obras, demandadas largo tiempo por los habitantes del barrio y, como veremos más adelante, uno de los ejes reivindicativos del movimiento social, rápidamente fueron puestas al servicio de la acumulación del capital a través del mercado inmobiliario, llevando incluso a nuevos procesos de desalojo y segregación espacial para estos pobladores.

Estos procesos guardan correlación con la tendencia señalada por Janoschka y Sequera (2014) hacia las "políticas de desplazamiento" de las clases populares de las áreas centrales de las ciudades. Aún cuando no se trata de un área central de la ciudad en términos geográficos, creemos que el planteo de los autores resulta esclarecedor al iluminar procesos de transformación urbana en áreas "centrales" en términos de valorización capitalista. La periferia semi-urbana de la ciudad de Rosario, así como las zonas aledañas al río destinadas anteriormente a las actividades portuarias fueron por décadas espacios de urbanización informal por parte de los sectores populares. Pero 
recientemente se convirtieron en el foco de emprendimientos inmobiliarios que destacan una privilegiada relación con el medio ambiente - clubes de campo, barrios privados, "puertos" comerciales y habitacionales - exclusiva para las capas medias y altas de la sociedad.

En el seno de este proceso de destrucción y creación, en el que la ciudad se rehace, como apunta Harvey (2014), la economía de acumulación de riqueza se transforma violentamente en economía de desposesión. De este modo, el autor señala: "la absorción del excedente mediante la transformación urbana tiene empero un aspecto aun más tenebroso: ha supuesto repetidas rachas de reestructuración urbana mediante una "destrucción creativa" que casi siempre tiene una dimensión de clase, ya que suelen ser los más pobres y menos privilegiados, los marginados del poder político, los que más sufren en esos procesos. Para hacer surgir la nueva geografía urbana del derrumbe de la antigua se requiere siempre violencia" (2014: 37).

Cabe destacar, a su vez, que la "destrucción creativa" (Harvey, 2014) ocurre no solo impulsada por la especulación inmobiliaria, sino que está sedimentada en el interés político que orienta los procesos de reordenamiento urbano en la ciudad. Por motivos justificados por el gobierno municipal en un "error de coloreo", el mapa urbano mostraba una doble identificación para el área a la que estamos aludiendo: como "zona no urbanizable", y como futuro emprendimiento privado, dando cuenta del conocimiento previo acerca del proyecto de urbanización existente ${ }^{13}$. Lo que es más, el vínculo entre el Estado local y el grupo inversor se volvería palpable a través del accionar de ciertos ediles que, en plena escalada del conflicto que habría de suscitarse, recorrían el área acompañados de representantes de la empresa privada para constatar la ocupación de los terrenos ${ }^{14}$.

\title{
Ante a las topadoras ${ }^{15}$, escuelas: la creación de la experiencia educativa
}

\begin{abstract}
Algo cambió en la forma de ver a nuestros territorios rebeldes. Pudimos constatar que la desidia a la que arrojaron a los territorios quienes decidieron sacarse esta disputa de encima, sumado a la capacidad de las corporaciones para dominar el espacio y producirlo, daba como resultado la necesidad urgente de acelerar la creación de una nueva geografía política" (Documento del movimiento social, año 2011).
\end{abstract}

\footnotetext{
13 (Documento de difusión del movimiento social, año 2008).

${ }^{14}$ R35. Observación en protesta del movimiento social. Fecha: 27/09/2011).

15 En Argentina, el término "topadora" designa una máquina de gran porte que se utiliza para excavar. Metafóricamente, se emplea el término para aludir a la acción de derribar o desalojar viviendas u otras edificaciones.
} 
Como ya señalamos, en décadas recientes las ciudades se han convertido también en escenario de luchas políticas, tomando como reivindicación central el derecho a la ciudad (Harvey, 2014).

El trabajo de Holston (2009) propone la categoría de "ciudadanía urbana" para considerar procesos de movilización que tienen por base la residencia urbana y el derecho a la ciudad como reivindicación. El autor apunta a mostrar el modo en que las dificultades materiales y legales de la autoconstrucción de la residencia a la que fueron empujados los pobres urbanos en Brasil, fueron la base de procesos de politización y movilización. Sostiene, entonces, que no es "dentro de la plaza cívica donde los pobres urbanos articulan sus demandas con más fuerza y originalidad sino más bien en el dominio de la vida cotidiana y doméstica, que toma forma en las remotas periferias urbanas en torno a la construcción de la residencia" (2009: 46).

Las movilizaciones por el derecho a consumir la ciudad y vivir en condiciones dignas se denominaron desde la teoría social "movimientos sociales urbanos", y definidas principalmente a partir de los aportes de Manuel Castells (1987). Desde esta perspectiva, siendo la ciudad concebida como ámbito exclusivo del consumo colectivo, los movimientos urbanos quedaron circunscriptos al problema de la reproducción, y si bien innovadores en sus métodos - principalmente por fuera de los canales institucionales-, serían solo defensivos, sin capacidad de realizar transformaciones estructurales. En este sentido, se los consideró "nuevos" movimientos sociales, por contraposición a los movimientos clásicos, ligados a las clases y a los partidos políticos, vinculados a la esfera de la producción. Sin embargo, algunas perspectivas criticaron la separación tajante entre producción y reproducción, ya que a nivel empírico las luchas en ambas esferas a menudo convergen: "no hay lucha de clases que no sea a la vez una lucha por las condiciones reproductivas de la clase obrera; por ende, tampoco hay lucha por mejores condiciones de vida que no sea parte de la lucha de clases" (Evers y otros, 1982: 718).

Es así que algunas investigaciones locales han apuntado a mostrar, a partir del análisis de casos concretos, cómo pueden volverse "porosas" las distinciones históricas entre producción y reproducción (Manzano, 2016). Desde este enfoque, Manzano (2016), ha apuntado a mostrar la producción por parte de los movimientos sociales de empleo y bienes urbanos, en tanto que bienes comunes, a partir del trabajo colectivo movilizado. 
La actividad del movimiento social que más tarde impulsaría la experiencia educativa foco de nuestra investigación, se inicia en 2005. Se trataba de un grupo de jóvenes estudiantes universitarios - varones y mujeres - que, en un primer momento, se acercaron al barrio para desarrollar actividades educativas con niños:

\begin{abstract}
Empezamos con apoyo escolar, en lo de una vecina que nos prestó la casa para eso. Charlando con los padres de los chicos nos empezamos a enterar los problemas del barrio, los desalojos, las inundaciones. Empezamos a trabajar, y se formaron las asambleas, los representantes por manzana, etcétera" (R1. Militante-docente. Entrevista. Fecha: 15/05/2010).
\end{abstract}

Fueron las demandas en torno a las inundaciones, y especialmente una que resultó crítica, en el año 2007, lo que dio fuerza a la incipiente organización. El recientemente conformado movimiento social se ocupó de censar a los habitantes para relevar daños y pérdidas materiales durante la inundación, y posteriormente, demandó al Estado municipal la entrega de un resarcimiento económico en función de los datos obtenidos. La posterior obtención de ese resarcimiento se convirtió en un hecho fundante que marcaría la recordada "primer victoria" del movimiento social.

Pronto a las luchas que demandaban la intervención del Estado municipal frente al problema de las inundaciones se sucedieron las que ponían en escena el uso del suelo y la apropiación de excedente urbano a través de la especulación inmobiliaria. Es que cuando las tan ansiadas obras sobre el canal que atraviesa el barrio fueron anunciadas, quedó al desnudo el proyecto de creación de un barrio privado, que comenzaba a ejecutarse. Los "desalojos silenciosos"16 que ya había comenzado a denunciar el movimiento social crecieron en intensidad y adquirieron visibilidad pública. Ante los intentos por parte del gobierno municipal de llevar adelante un "desalojo asistencial" (Gago y Pérez, 2014) que implicara la relocalización de las familias en otros barrios, el movimiento social puso en marcha distintas estrategias.

En primer lugar, la organización desarrolló una política activa de ocupación de espacios en la zona en conflicto, especialmente a través del impulso de distintos proyectos educativos y culturales: apoyo escolar para niños, radio comunitaria para niños y jóvenes, talleres de informática, entre otros; así como productivos: huertas comunitarias, una cooperativa textil, y el más importante en relación a las dimensiones

\footnotetext{
${ }^{16}$ El movimiento social denunciaba durante los años 2007 y 2008 la existencia de "desalojos silenciosos", es decir, acciones de parte de sectores privados, en pequeña escala y a menudo invisibilizadas, que por la fuerza (ya sea mediante extorsión o violencia directa) buscaban remover a las familias que ocupaban la zona (Documento de difusión del movimiento social, año 2008).
} 
que adquirió, un tambo y fábrica de productos lácteos. Esta ocupación del territorio se concibió como estrategia para detener al avance de los desalojos en la totalidad de la zona rural del barrio:

El tambo para nosotros es una especie de muralla, digamos, el que barre con el tambo barre con el resto de las 250 ha. Y sostener el tambo ahí es defender el resto de las 250 ha. Entonces, (...) está bueno poder pensarlo como eso, como un símbolo que en realidad sostiene o no la privatización del resto de las ha. de la zona rural del barrio (R34. Militante-docente. Entrevista. Fecha: 24/05/13).

En segundo lugar, el movimiento social diseñó proyectos que aspiraban a intervenir en mayor o menor grado sobre la planificación urbana, cuya aplicación intentó, con mayor o menor éxito, consensuar con el gobierno de la ciudad ${ }^{17}$. Uno de ellos fue el "plan de seguridad territorial": un proyecto presentado por el movimiento social en el año 2011 como modo de acordar una intervención estatal sobre el barrio, de acuerdo con las prioridades definidas por la organización. El acuerdo establecía la "inversión pública para la producción y el desarrollo" e "intervenciones para el mejoramiento de los espacios comunitarios y la vía pública", a través del aporte gubernamental de "canastas de materiales, herramientas e insumos" y "la mano de obra y la ejecución" del movimiento social" (Documento de difusión del movimiento social, año 2011).

Como ya señalamos, nos interesa enfocar estos procesos desde la creación, en 2011, de una escuela para jóvenes y adultos en el corazón del área en disputa. A partir de la definición de poner en marcha el proyecto, desde el movimiento social se convocó a los futuros docentes: un grupo heterogéneo de 16 personas, compuesto mayormente por estudiantes avanzados de carreras universitarias o terciarias, así como también docentes en ejercicio o próximos a su jubilación. El plantel se completó, a su vez, con algunos militantes de la organización que tomaron a su cargo la coordinación del espacio educativo $^{18}$. A diferencia de este cuerpo docente, cuyos integrantes provenían

\footnotetext{
${ }^{17}$ Durante esta etapa, la única propuesta a escala de la ciudad fue la presentación de un proyecto de ley para prohibir los barrios privados que, contando con el apoyo de ediles de otras fuerzas políticas, fue finalmente aprobado en el año 2010 (R11. Observación en sesión del Concejo Deliberante. Fecha: 02/12/2010). El proyecto político se consolidó durante esos años en torno a un territorio específico y a sus problemáticas, haciendo de la ocupación y producción del espacio barrial la principal estrategia de lucha.

${ }^{18}$ Nos parece pertinente introducir una aclaración. Por "militantes", nos referimos, retomando una categoría social, a aquellos que integran el movimiento social de forma orgánica. Para diferenciar a los docentes que se incorporan en calidad de tales, de aquellos que ya formaban parte orgánica del movimiento social con anterioridad y por ello se integran a la experiencia educativa, denominaremos
} 
de distintos barrios de la ciudad, el estudiantado se conformó exclusivamente con habitantes del barrio, jóvenes y adultos que podían o no formar parte de la organización, ya que esto no resultaba un requisito para su incorporación. Inicialmente se alcanzó un número aproximado de 25 estudiantes, que fue, no obstante, oscilando con el transcurso del tiempo.

La creación de la escuela se constituyó, en primera medida, en reivindicación del derecho a la educación como parte de los consumos colectivos que hacen posible la reproducción de la vida social. Al presentar la experiencia educativa en un acto inaugural, uno de los futuros docentes señalaba:

Somos acérrimos defensores de la educación pública. Para nosotros la educación es pública, o no es. Y en ese sentido, empezar a pensar que la educación puede ser pública y comunitaria, pública y autogestiva, pública y colectiva, me parece que son los desafíos que tiene esta escuela (R12. Docente. Inauguración de la escuela. Fecha: 04/04/2011).

Pero a su vez, la importancia de la propuesta educativa era puesta claramente en clave de la lucha territorial, al declamar, orgullosos: "ante las topadoras, escuelas" (R12/2011. Inauguración de la escuela. Militante del movimiento social y docente. Fecha: 04/04/2011), Como frecuentemente argumentaban los militantes de la organización, confiaban en que la existencia de una escuela sería un freno mucho más efectivo ante los intentos de desalojo:

Nosotros consideramos que una escuela en el barrio, hace a la seguridad de ese territorio. No es lo mismo tumbar una casa de un vecino, que tumbar una escuela, una radio, una huerta, que tumbar casas y nada más (R12. Militante-docente. Observación en la inauguración de la escuela. Fecha: 04/04/11).

Lo que es más, al mismo tiempo que permitía ocupar activamente terrenos en el corazón del área que estaba siendo desalojada, la creación de la escuela habilitó al movimiento social a demandar al Estado municipal la provisión de otros servicios hasta el momento ausentes en la "zona rural". Bajo el argumento de la existencia de la nueva escuela, la organización logró ejercer la presión necesaria para la realización de obras como la extensión del tendido eléctrico y el mejorado de las calles - que mejoraron las condiciones de vida de los habitantes de la zona.

“militantes-docentes" a estos últimos. Estas distinciones no son estáticas, pero las presentamos a los fines de orientar al lector. 
¿Cómo podemos comprender el lugar relevante que tomó la experiencia educativa dentro de la lucha territorial? Si bien la organización había desarrollado anteriormente otros espacios educativos en el barrio, la nueva escuela adquiría características especiales al incorporarse en un campo configurado de modo reciente por los "Bachilleratos Populares" a nivel nacional. Aunque no podemos abordar aquí la totalidad de estas características, lo que nos interesa destacar es que estas escuelas se posicionaron argumentativamente como una alternativa frente al carácter excluyente del sistema educativo oficial, y desde allí reclamaron al Estado la posibilidad de otorgar títulos que certificaran el cumplimiento de la educación secundaria para sus egresados, al igual que cualquier establecimiento educativo oficial. Como señalan Manzano y Ramos, sobre la base de los repertorios que orientan las prácticas de movilización, reivindicación y/o demanda "no sólo se están presuponiendo, impugnando o resignificando ideas de Estado, sino también se está encarnando, bifurcando, reemplazando o ampliando críticamente su operatoria como sistema" (2015: 13).

Retomamos, en este punto, la interpretación que propone Roseberry (1994), al concebir los procesos hegemónicos como un marco material y cultural común, que es en parte discursivo: "un lenguaje común o forma de hablar sobre las relaciones sociales que demarca los términos centrales en torno a y en función de los cuales se dan la controversia y la lucha" (1994: 127). De este modo, un orden dominante establece los procedimientos legítimos, las formas adecuadas para expresar aceptación o descontento, y moldea, dentro de un campo de fuerza dinámico y multidimensional, tanto al Estado como a la "cultura popular" (Roseberry, 1994).

Sostenemos, entonces, que al establecer la ocupación de tierras como un "hacer escuela", inscribiéndose así en el campo configurado por la aparición de los "Bachilleratos Populares", el movimiento social estaba colocando la lucha por el espacio urbano en un lenguaje adecuado para ser oído, y al mismo tiempo redefiniendo sobre qué ejes se plantearía la disputa (Roseberry, 1994).

Es así que la creación de la experiencia educativa no solo ponía en agenda el derecho a acceder a la educación como consumo colectivo, sino también como posibilidad de construcción colectiva al "hacer escuela". En este sentido, resulta elocuente que no se trataba de demandar al estado la creación de escuelas públicas estatales en las periferias de la ciudad sino de reclamar para sí el derecho a producir 
escuelas y con ello, el conjunto de relaciones sociales que las mismas permiten desplegar en la trama barrial.

De este modo, entendemos que la práctica política del movimiento social no se agota en la reivindicación del derecho a consumir la ciudad, sino también, fundamentalmente, a producirla. Retomando las palabras de Harvey, podemos reconocer que "el derecho a la ciudad es por tanto mucho más que un derecho de acceso individual o colectivo a los recursos que esta almacena o protege; es un derecho a cambiar y reinventar la ciudad de acuerdo con nuestros deseos. Es, además, un derecho mas colectivo que individual, ya que la reinvención de la ciudad depende inevitablemente del ejercicio de un poder colectivo sobre el proceso de urbanización" (2014: 20).

Podemos sostener que, de este modo, el movimiento social avanza en la producción de lo común. De acuerdo a Harvey (2014), mientras lo público ha sido siempre objeto de control estatal y fuente de desarrollo capitalista, lo común refiere a una relación colectiva y no mercantilizada con espacios y bienes que se protegen y mejoran para el beneficio mutuo. Lo común requiere, a la vez, una acción política de apropiación $^{19}$. Así, la creación de la escuela del movimiento social supone, a partir del trabajo colectivo, la apropiación de la educación ${ }^{20}$ como bien público para hacer de ella un bien común, y en este "hacer escuela", reclamar el derecho a reinventar la ciudad (Harvey, 2014).

Estos procesos, sin embargo, son ambivalentes y no se desarrollan sin contradicciones. La búsqueda de reconocimiento oficial de la escuela abrió un campo de negociaciones en el que el proyecto debió adaptarse a ciertos requerimientos estatales, tales como el abandono del terreno ocupado y la relocalización del edificio escolar en una casa alquilada, alejada del área de conflicto. Estas tensiones se profundizaron, a su vez, con la posterior conformación de un partido político con tres concejales electos en la última votación (año 2015), que reemplazó el modo anterior de organización de tipo

\footnotetext{
${ }^{19}$ Ahora bien, lo común no supone, en el sentido que lo estamos tomando, la ausencia de jerarquías ni de límites, o la "dispersión de iniciativas y la descentralización de actos" que distingue Stavrides (2016: 240). En la experiencia desarrollada por el movimiento social, la conformación de lo común se concretiza atravesada por ciertos niveles de jerarquía y regulación del espacio bajo cierta direccionalidad e intencionalidad política.

${ }^{20}$ Retomamos las palabras de Harvey entendiendo que refieren a un sentido restringido de "educación", como equivalente a escuela o sistema escolar, mientras que la educación en sentido amplio no podría pensarse acotada al dominio de lo público.
} 
movimentista y canalizó la contienda por las vías institucionales del Estado. A la par, proyectó el espacio de construcción política a escala de la ciudad.

Si bien estas transformaciones quedan por fuera del período que delimitamos para nuestro análisis, permiten poner a la luz algunas de las tensiones que se hallan en la base de los procesos abordados, cuyo estudio deberá ser profundizado a partir de futuras indagaciones.

\section{Palabras finales}

En este trabajo nos propusimos presentar algunas reflexiones a partir de la conformación de un "Bachillerato Popular" como parte del proyecto político de un movimiento social, cuyo accionar se inscribe en un entramado barrial de pobreza urbana y desigualdad social de la ciudad de Rosario. En particular, intentamos avanzar en una línea de análisis que nos permitiera repensar este proceso a la luz de las formulaciones acerca del derecho a la ciudad.

Al considerar los procesos que fueron conformando la periferia de la ciudad de Rosario y, en particular, el barrio en el cual se inscribe el accionar de la organización, apuntamos a destacar el desplazamiento y la desposesión de los sectores populares como la contracara de la apropiación de excedente urbano, a partir de proyectos de transformación y desarrollo de la ciudad. A la vez, nos propusimos superar miradas excluyentes acerca de los procesos de producción y reproducción, retomando aportes que nos ayuden a pensar los lugares de residencia de las clases populares como sitios fértiles para la construcción política (Holston, 2009; Manzano, 2016).

De este modo, nos propusimos analizar la creación de la experiencia educativa como parte de las luchas que impulsa el movimiento social, comprendiendo a las mismas como reivindicación del derecho a la ciudad. Se trata de luchas por el derecho a consumir y producir la ciudad y que, en tal sentido, desbordan las demandas ligadas exclusivamente a lo educativo. Como señala Harvey, el derecho a la ciudad así entendido "supone reivindicar algún tipo de poder configurador del proceso de urbanización, sobre la forma en que se hacen y rehacen nuestras ciudades" (2014: 21).

Desde esta perspectiva, se puede sostener que la escuela del movimiento social se constituyó como estrategia de ocupación de terrenos frente a la posibilidad de expropiación, al mismo tiempo que permitía reclamar la provisión de servicios urbanos 
al Estado municipal. De igual manera, se puede comprender como una apropiación de un bien público, la educación escolar, para hacer de ella un bien común (Harvey, 2014).

En síntesis, la creación de la escuela puede ser vista como parte de las estrategias desplegadas por la organización política que permitieron cuestionar lógicas dominantes en la configuración de la ciudad, y avanzar en la producción colectiva de lo urbano.

\section{Referencias}

AMPUDIA, Marina (2013). Educación y Autogestión. Estética Política y Autogestión. Estética política de los Bachilleratos Populares. En R. Elisalde, M. Dal Ri Neusa, M. Ampudia, \& A. Falero, Movimientos sociales, educación popular y trabajo autogestionado en el Cono Sur (págs. 31-48). Buenos Aires : Buenos Libros.

BERNADI, Gabriela; SANCHEZ, Silvana (2006) Procesos de concentración espacial de la pobreza urbana. Retrato de una configuración. En: Achilli, et al. Memorias y experiencias urbanas. (2006) 1a ed. Rosario. Universidad Nacional de Rosario.

BRENNER, Neil y Nik Theodore (2002) "Cities and Geographies of Actually Existing Neoliberalism”. Antipode 34 (3): 349-379. 77//

BROGUET, Julia. (2016) "Lo negro en algún lado está...": orden espacial-racial y candombe afrouruguayo en el barrio Refinería (Rosario, Argentina). Revista colombiana de antropología. Vol. 52, No1 Enero-junio 2016. PP197-222

CAISSO, Lucía. (2014) "Una escuela como ésta. Experiencias educativas en un movimiento social de la ciudad de Córdoba (Argentina)". Tesis doctoral. Inédita.

CÁMPORA, Edith; CASELlA, Mariano; LLOVERA, Soledad; GIL, Mariano; SERRA, Julia; (2017) "Re-problematizando el concepto de juventudes: entrecruzando definiciones y abordajes”. En: Romero Acuña, M.; Torres Leal, E. (comps) Avances de investigación. Aportes y dificultades. $1^{\mathrm{a}}$ ed. - Rosario : UNR Editora. Editorial de la Universidad Nacional de Rosario. CEACU- Centro de Estudios Antropológicos en Contextos Urbanos.

CÁMPORA, Edith; GIAMPANI, Leandro (2006) La ciudad y sus territorios: sentidos sociales en juego. En: Achilli, et al. Memorias y experiencias urbanas. (2006) 1a ed. Rosario. Universidad Nacional de Rosario.

CARNELLI, Lucía, RUBINSZTAIN, Paola, SAID, Shirly (2015). Una década de Bachilleratos Populares de Jóvenes y Adultos en la Argentina: Aportes para un análisis cuantitativo a partir del Primer Relevamiento Nacional. Buenos Aires, MIMEO.

CASTELLS, Manuel. (1987): Movimientos sociales urbanos. Siglo XXI, México; 9-18 y conclusiones.

CRAVINO, María Cristina, PALOMBI, Ariel, QUINTAR, Aida (2014) "Derecho a la ciudad y conflictos urbanos. Lo que dejó la toma del parque Indoamericano". En: Cravino (org) Derecho a la ciudad y conflictos urbanos. La ocupación del Parque Indoamericano. UNGS. Los Polvorines.

DE MARCO (h.) Miguel A. (2016) De los orígenes y los barrios de Rosario (tercera entrega) - La expansión al oeste. Revista de la Bolsa de Comercio de Rosario. Septiembre de 2016. Año CV / N. 1529

ELISALDE, Roberto (2008). Movimientos sociales y educación: Bachilleratos Populares en empresas recuperadas y organizaciones sociales. Experiencias pedagógicas en el campo de la educación de jóvenes y adultos. En R. Elisalde, \& M. Ampudia, 
Movimientos sociales y educación. Teoría e historia de la Educación Popular en Argentina y América Latina (págs. 65-102). Buenos Aires: Buenos Libros.

EVERS, Tilman; MÜLLER-PLANTENBERG, Clarita; SEPESSART, Stefanie (1982) Movimientos barriales y Estado: luchas en la esfera de la reproducción en América Latina. En: Revista Mexicana de Sociología, 2, México; 703-756. En: http://www.jstor.org/stable/3540284?seq=11\#page_scan_tab_contents

GAGO, Verónica y GARCÍA PÉREZ, Eva (2014). "Ciudad próspera, ciudad monstruosa: nuevas racionalidades urbanas a partir del caso Indoamericano". En: Quid 16. $\mathrm{N}^{\circ} 4, \mathrm{pp}, 66-83$.

GARCÍA, Javier (2011) “Aprendiendo a hacer escuelas. Las complejas y dinámicas relaciones entre Bachilleratos Populares y Estado. Tesis de Maestría. Inédita.

GLUZ, Nora (2013) Las luchas populares por el derecho a la educación: experiencias educativas de movimientos sociales. Buenos Aires. CLACSO

GRAVANO, Ariel (2013) Antropología de lo urbano. $1^{\mathrm{a}}$ ed. $1^{\mathrm{a}}$ reimp - Tandil: Universidad del Centro de la Provincia de Buenos Aires.

GRAVANO, Ariel (2014). Antropología de ciudades medias: tres hipótesis sobre la relación entre sistema urbano e imaginarios. XI Congreso Argentino de Antropología Social. Rosario, Argentina.

HARVEY, David. (2014) Del derecho a la ciudad a la revolución urbana. Akal. Argentina.

HOLSTON James. (2009) "La ciudadanía insurgente en una era de periferias urbanas globales. Un estudio sobre la innovación democrática, la violencia y la justicia en Brasil.” En: De la Matta, Gabriela (ed.) Movilizaciones sociales ¿nuevas ciudadanías? Reclamos, derechos, Estado en Argentina, Bolivia y Brasil. Editorial Biblos. Buenos Aires.

JANOSCHKA, Michael; SEQUERA, Jorge. (2014) "Procesos de gentrificación y desplazamiento en América Latina, una perspectiva comparativista". Contested Cities

LANGER, Eduardo (2011). Prácticas discursivas y dificultades en el hacer de estudiantes y docentes en un bachillerato popular : tensiones y alcances en la producción de resistencia desde un dispositivo pedagógico emergente. Tesis de Maestría. FLACSO. Sede Académica Argentina, Buenos Aires.

MANZANO, Virginia (2016) "Urbanización, trabajo y políticas de la vida (colectiva): El movimiento Tupac Amaru en ciudades del norte argentino". En: Actas Congreso Internacional Contested Cities- Madrid, España.

MANZANO, Virginia; RAMOS, Ana M. (2015) “Introducción. Procesos de movilización y de demandas colectivas: estudios y modos de abordar 'lo político' en la vida social" En: Identidades. Núm. 8, Año 5, pp. 01-25

MOCTEZUMA MENDOZA, Vicente (2016) "El desplazamiento de lo posible: experiencia popular y gentrificación en el Centro Histórico de Ciudad de México". En: Íconos. Revista de Ciencias Sociales. Num. 56 Quito, septiembre 2016, pp. 83-102. Facultad Latinoamericana de Ciencias Sociales-Sede Académica de Ecuador.

NEMCOVSKY, Mariana (comp) (2004) Los Barrios en la Memoria. Talleres con adultos mayores. Escuela de gerontología. Secretaría de Promoción Social. Municipalidad de Rosario.

ROSEBERRY, William (1994) Hegemonía y el lenguaje de la controversia. En: Joseph Gilbert y Daniel Nugent Everyday Forms os State Formation: Revolution and the Negotiation of Rule in Modern Mexico. Duke University Press. Traducción Hernando Calla Ortega.

SANTILLÁN Laura (2015). La demanda por la educación en barrios populares del Gran Buenos Aires: acerca de la militancia política, el voluntariado social y la actuación 
colectiva de los pobladores (1985 y 2015). XI Reunión de Antropología del Mercosur. Montevideo, Uruguay.

SILVA, Ana (2016) Espacios culturales en el Barrio de La Estación de Tandil. Memorias y tramas en la producción de lo público de una ciudad media. AURA. Revista de Historia y Teoría del Arte. ISSN: 2347-0135 - No4 - Septiembre 2016 (Pp. 128-149) SINGER, Paul (1981) Economía política de la urbanización. Siglo XXI, México; 7-17. STAVRIDES, Stavros. (2016) "Hacia la ciudad de Umbrales". Apéndice. "Plazas en movimiento" (pp. 233-248)

VERA, Paula (2012) Disputas en la construcción simbólica de las ciudades. El caso Rosario. La Trama de la Comunicación, Volumen 16. UNR Editora, 2012 (pp. 69-86)

VERA, Paula (2013) Imaginarios Urbanos y procesos de urbanización en las nuevas ciudades turísticas. El caso de la ciudad de Rosario, Argentina. Bitácora 22 (1) 153-162. Universidad Nacional de Colombia. Bogotá.

VERA, Paula; ROLDÁN, Diego P. Y PASCUAL, Cecilia M. (2016) La construcción escenográfica de la ciudad. Espacio público, turismo e itinerarios en Rosario (Argentina) Chasqui. Revista Latinoamericana de Comunicación N. ${ }^{\circ}$ 130, diciembre 2015 - marzo 2016 (Sección Monográfico, pp. 199-219) ISSN 1390-1079 / e-ISSN $1390-924 \mathrm{X}$

Recebido em: 16/10/2017. Aprovado em: 15/11/2017. 